\title{
oints
}

$\sqrt{2}$

\section{Degenerative disease in rotator cuff tears: what are the biochemical and histological changes?}

\author{
SILVANA DE GIORGI ${ }^{1}$, MICHELE SARACINO ${ }^{1}$, ALESSANDRO CASTAGNA $^{2}$ \\ ${ }^{1}$ Department of Basic Medical Sciences, Neurosciences and Sensory Organs, University of Bari, Italy \\ ${ }^{2}$ Shoulder Service, IRCCS Humanitas Institute, Rozzano, Milano, Italy
}

\begin{abstract}
The histopathological changes associated with rotator cuff tears include thinning and disorganization of collagen fibers, the presence of granulation tissue, increased levels of glycosaminoglycans, fibrocartilaginous metaplasia, calcification, fatty infiltration, and necrosis of the tendon margin with cell apoptosis. The biochemical changes include an increase in the expression of matrix metalloproteinases (MMPs) and a decrease in tissue inhibitor of metalloproteinases (TIMP) messenger ribonucleic acid expression. Histological evidence of tendinopathy has been found in patients with rotator cuff tear. Biochemical changes include significant increases in MMP1, MMP2, MMP3, and in TIMP1 and TIMP2 levels, not only at the lateral supraspinatus edge, but also in the macroscopically intact portion of the supraspinatus tendon and in the intact subscapularis.

The tissue in the ruptured area of the supraspinatus tendon undergoes marked rearrangement at molecular levels. This involves the activity of MMP1, 2, and 3 and supports a critical role of MMPs in tendon physiology. Intact parts of the torn supraspinatus tendon can present the histopathological changes associated with rotator cuff tears. These findings suggest that biochemical changes can already occur in a macroscopically intact tendon and seem to point to a global degenerative process in the shoulder.
\end{abstract}

\footnotetext{
Corresponding Author:

Silvana De Giorgi, MD

Department of Basic Medical Sciences, Neurosciences and

Sensory Organs, University of Bari

Via Murge 59/A, 70124 Bari, Italy

Phone /Fax: +39 080559720/0805592721

E-mail: silvana.degiorgi@uniba.it
}

Key Words: biology, histology, degenerative changes, rotator cuff tear.

Rotator cuff tears are a frequent cause of shoulder pain and disability. The pathogenesis and the biochemical changes associated with rotator cuff tears are unclear, but they may arise from a combination of extrinsic impingement and intrinsic alterations within the tendon tissue itself (1). The etiology of rotator cuff disease is likely multifactorial, including age-related degeneration and microtrauma. Smoking, hypercholesterolemia and genetics have all been shown to influence the development of rotator cuff tearing (2).

Tendons have a water content of $70 \%$, while type I collagen accounts for $85 \%$ of their dry weight. Cells are scarce and those present are mostly (90-95\%) fibroblasts. The histopathological changes associated with rotator cuff tears include thinning and disorganization of collagen fibers, the presence of granulation tissue, infiltration of glycosaminoglycans, fibrocartilaginous metaplasia, calcification, fatty degeneration, and necrosis of the tendon margin with cell apoptosis (3-5). These changes are also present in macroscopically intact tendons in the early stage of the degenerative process $(3,6)$. The degenerative changes at the tendon margin can also explain the high rate of recurrence after surgery, even in cases with less than grade 2 fatty infiltration (4). Chronic rotator cuff tears in elderly patients have limited healing potential and a high risk of recurrence, even if surgically treated. In these cases, the weak healing process seems to be produced more from the bursal side than from the tendinous one. Conversely, the healing potential of small and acute rotator cuff tears, in young people, has been seen to be better, thanks to low rates of apoptosis and fibrocartilaginous metaplasia and high levels of neoangiogenesis (3). 
Necrotic tendon tissue, fibrin and fibronectin appear to be signs of tendon degeneration, while fibrosis and thinning of fascicles have also been found in controls without rotator cuff tears (7).

The biochemical changes in rotator cuff tears are yet not perfectly known. Matrix metalloproteinases (MMPs), which are a family of 24 zinc-dependent endopeptidases, play an important role in tissue remodeling after injury, but an excess of their activity can lead to progressive weakening of the extracellular matrix (ECM) of tendons. The collagenases (MMP1, MMP8, and MMP13) are able to degrade the collagen fibrils that confer mechanical strength on the tissue. The gelatinases (MMP2 and MMP9) degrade smaller collagen fragments released during the activity of the collagenases. The activity of endogenous MMPs is inhibited by endogenous tissue inhibitors of MMPs (TIMPs) and the relative balance between MMPs and TIMPs plays an essential role in tendon development, morphogenesis and normal remodeling (8). Studies in pathologic tendons have demonstrated an increase in the expression of MMPs and a decrease in TIMP mRNA expression in tenocytes (9-11).

Castagna et al. (12), in a study of 13 patients, found that levels of MMPs 1, 2, and 3 were altered not only at the edge of the torn supraspinatus tendon, but also in areas far from the lesion and in the arthroscopically and histologically healthy subscapularis tendon. This suggests that biochemical changes, such as an increase in MMPs, can already occur in macroscopically intact tendon. There were no significant differences in MMP and TIMP values between the supraspinatus tendon tear area, the medial macroscopically healthy area of the supraspinatus tendon and the subscapularis tendon used as a control. The levels of MMPs 1, 2, and 3 and TIMPs 1 and 2 were significantly greater at the lateral edge of the torn supraspinatus tendon, as well as in its intact medial portion and in the healthy subscapularis tendons, compared with the levels of MMPs $8,9,10$, and 13. No significant differences in the levels of TIMPs 1 and 2 were found between the specimens assessed. These findings seem to point to a global degenerative process in the joint. These changes could be the pathogenic precursor of a subsequent tear or a tendinopathic process occurring in the rupture area and extending to the portion of the tendon medial to the site of rupture. Lakemeier et al. (13) showed that MMPs 1, 3, and 9 were also increased in degenerated long head of the biceps tendon in the presence of rotator cuff tears.

TIMPs 1, 2, and 4 are present in the tissue matrix and in the blood, while TIMP3 is present only in the ECM. TIMPs have several functions. They can inhibit MMPs and regulate angiogenesis and cellular growth (14). The balance between MMPs and TIMPs plays a crucial role in tendon development, morphogenesis and remodeling (15). In tendinopathy with chronic rotator cuff tear, MMPs are increased and TIMPs are decreased (16), while in small rotator cuff tears and in young people both MMPs and TIMPs are increased. Tajana et al. (17) demonstrated a correlation between MMP2 and MMP9 in the synovial fluid and the severity of rotator cuff disease. TIMP1 may be upregulated in acute tears as an early marker of ECM remodeling (18).

It has been shown that tendon tissue can actively secrete pro-collagenase, an enzyme that, once activated, is capable of remodeling collagen, the major connective tissue component of tendon. Collagenase was produced even in unstimulated cultures, although the concentrations of TIMPs were usually greater than those of collagenase in most samples. Some activation of collagenase appeared to have occurred. These results indicate that tendon tissue cells are capable of producing a remodeling response even in end-stage tendon disease (9).

Recent studies suggest that MMPs could play a role in the inflammatory process through cytokines such as IL $1,4,6$, and 10, TNF, and GF, which can increase the synthesis and release of neoepitopes from the ECM (19). Inflammatory cytokines are involved in pain and tendon healing. Pain in tendinopathy could be mediated by substance $\mathrm{P}(\mathrm{SP})$, a neurotransmitter, which can modulate the genic expression of MMPs and TIMPs in fibroblasts. SP is implicated in the healing process and, when administered exogenously, appears to increase fibroblastic proliferation and tendon healing. Patients who develop a frozen shoulder after a rotator cuff repair have higher plasma levels of SP than patients with a good postoperative outcome (20).

High levels of inflammatory cytokines, metalloproteases (MMP1 and MMP9), and cyclooxygenases, known to produce a catabolic environment, are present in the subacromial bursa of patients with rotator cuff tear, 
emphasizing the importance of subacromial bursectomy to reduce inflammation in cuff tears $(21,22)$. Some experimental studies have shown that the mechanical properties of tendons can be altered by the use of MMP inhibitors, such as certain antibiotics (e.g. doxycycline) and some bisphosphonates. MMP inhibitors, additionally, could help to increase the strength of the tendon repair. Indeed, sutures coated in doxycycline model have been successfully tested in a rat model (23).

After acromioplasty, higher levels of growth factors (GFs), such as PDGF, TGF-b1, and EGF, have been found in the subacromial bursa than in the peripheral blood. These molecules may have a role in the tendon healing process. Specifically, platelet-derived GFs have been shown to improve rotator cuff healing (24), even though more clinical trials are necessary to show the real efficacy of these platelet concentrates in tendon healing. Promising future clinical therapeutic applications could also derive from the presence of stem cells in rotator cuff and long head biceps tendons (25).

In conclusion, better knowledge of MMPs and TIMPs and more informed use of platelet concentrates and stem cells could open the way for a better strategy to optimize outcomes in rotator cuff tear therapy.

\section{References}

1. Garofalo R, Cesari E, Vinci E, et al. Role of metalloproteinases in rotator cuff tear. Sports Med Arthrosc. 2011; 19:207212.

2. Tashjian RZ. Epidemiology, natural history, and indications for treatment of rotator cuff tears. Clin Sports Med. 2012; 31:589-604.

3. Chillemi C, Petrozza V, Garro L, et al. Rotator cuff re-tear or non-healing: histopathological aspects and predictive factors. Knee Surg Sports Traumatol Arthrosc. 2011;19:1588-1596.

4. Goutallier D, Postel JM, Van Driessche S, et al. Histological lesions of supraspinatus tendons in full thickness tears of the rotator cuff. Rev Chir Orthop Reparatrice Appar Mot. 2005; 91:109-113.

5. Longo UG, Franceschi F, Ruzzini L, et al. Histopathology of the supraspinatus tendon in rotator cuff tears. Am J Sports Med. 2008;36:533-538.

6. Chillemi C, De Cupis V, Pacchiarotti A, et al. Secondary cuff tears: histopathological aspects. Rev Chir Orthop Reparatrice Appar Mot. 2005; 91(S8): 70.

7. Tillander B, Franzén L, Norlin R. Fibronectin, MMP-1 and histologic changes in rotator cuff disease. J Orthop Res. 2002; 20:1358-1364.

8. Magra M, Maffulli N. Matrix metalloproteases: a role in overuse tendinopathies. Br J Sports Med. 2005;39:789-791.
9. Dalton S, Cawston TE, Riley GP, et al. Human shoulder tendon biopsy samples in organ culture produce procollagenase and tissue inhibitor of metalloproteinases. Ann Rheum Dis. 1995;54:571-577.

10. Gardner K, Arnoczky SP, Caballero O, et al. The effect of stress deprivation and cyclic loading on the TIMP/MMP ratio in tendon cells: an in vitro experimental study. Disabil Rehabil. 2008;30:1523-1529.

11. Osawa T, Shinozaki T, Takagishi K. Multivariate analysis of biochemical markers in synovial fluid from the shoulder joint for diagnosis of rotator cuff tears. Rheumatol Int. 2005;25:436-441.

12. Castagna A, Cesari E, Gigante A, et al. Metalloproteases and their inhibitors are altered in both torn and intact rotator cuff tendons. Musculoskelet Surg. 2013; 97 (Suppl 1):39-47.

13. Lakemeier S, Schwuchow SA, Peterlein CD, et al. Expression of matrix metalloproteinases 1,3 and 9 in degenerated long head biceps tendon in the presence of rotator cuff tears: an immunohistological study. BMC Musculoskelet Disord. 2010;11:271.

14. Dolery CM, Libby P. Atherosclerosis and proteinase activation. Cardiovasc Res. 2006;69:625-635.

15. Lo IK, Marchuk LL, Hollinshead R, et al. Matrix metalloproteinases and tissue inhibitor of matrix metalloproteinase mRNA levels are specifically altered in torn rotator cuff tendons. Am J Sports Med. 2004;32:1223-1229.

16. Arnoczky SP, Lavagnino $M$, Egerbacher $M$, et al. Matrix metalloproteinase inhibitors prevent a decrease in the mechanical properties of stress-deprived tendons: an in-vitro experimental study. Am J Sports Med. 2007;35:763-769.

17. Tajana MS, Murena L, Valli F, et al. Correlations between biochemical markers in the synovial fluid and severity of rotator cuff disease. Chir Organi Mov. 2009; 93 (Suppl 1):41-48.

18. Choi HR, Kondo S, Hirose K, et al. Expression and enzymatic activity of MMP-2 during healing process of the acute supraspinatus tendon tear in rabbits. J Orthop Res. 2002;20:927-933.

19. Pearce WH, Shively VP. Abdominal aortic aneurysm as a complex multifactorial disease: interactions of polymorphisms of inflammatory genes, features of autoimmunity and current status of MMPs. Ann NY Acad Sci. 2006;1085:117132.

20. Franceschi F, Longo UG, Ruzzini L, et al. Circulating substance $\mathrm{P}$ levels and shoulder joint contracture after arthroscopic repair of the rotator cuff. $\mathrm{Br} J$ Sports Med. 2008; $42: 742-745$.

21. Voloshin I, Gelinas J, Maloney MD, et al. Proinflammatory cytokines and metalloproteases are expressed in the subacromial bursa in patients with rotator cuff disease. Arthroscopy. 2005;21:1076.e1-e9.

22. Blaine TA, Kim YS, Voloshin I, et al. The molecular pathophysiology of subacromial bursitis in rotator cuff disease. J Shoulder Elbow Surg. 2005;14 (1 Suppl):84S -89S.

23. Pasternak B, Missios A, Askendal A, et al. Doxycycline-coated sutures improve the suture-holding capacity of the rat Achilles tendon. Acta Orthop. 2007; 78: 680-686.

24. Zhang Q, Ge H, Zhou J, et al. Are platelet-rich products necessary during the arthroscopic repair of full-thickness rotator cuff tears: a meta-analysis. PLoS One. 2013;8:e69731.

25. Randelli P, Conforti E, Piccoli $\mathrm{M}$, et al. Isolation and characterization of 2 new human rotator cuff and long head of biceps tendon cells possessing stem cell-like self-renewal and multipotential differentiation capacity. Am J Sports Med. 2013;41:1653-1664. 